\title{
Modelling of Thermal Behavior N-Doped Silicon Resistor
}

\author{
Fouad Kerrour ${ }^{1}$, Ali Boukabache ${ }^{2}$, Patrick Pons ${ }^{2}$ \\ ${ }^{1}$ Modeling of Energy Renewable Devices and Nano-Metric Laboratory, University of Mentouri, Constantine, Algeria \\ ${ }^{2}$ Laboratory of Architecture and Systems Analysis, National Centre of Scientific Research, Toulouse, France \\ Email: f_kerrour@yahoo.fr
}

Received June 14, 2012; revised July 15, 2012; accepted August 16, 2012

\begin{abstract}
From the analysis of the frequently models of mobility used in the literature, we determine by an identification method the temperature coefficients $\alpha$ and $\beta$ of a silicon resistance doped with donor atoms. Their variations show a non linear dependence according to the doping and the existence of a minimal value at particular concentration. Moreover, the comparison between the obtained results and those of a P-type resistance shows that there is a strong similarity in their thermal behaviours, except for a particular couple of $\alpha$ and $\beta$.
\end{abstract}

Keywords: Silicon; TCRs; Mobility; Doping; Temperature

\section{Introduction}

The increasingly miniaturization and integration require, for the modeling engineers and manufacturers of integrated circuits, to improve the behavioural models of the electronic components. Among the latter, semiconductor resistors are used in all components either as passive elements of an electronic circuit or as a sensor (pressure, temperature, chemical species, etc.). In all cases, and in order to preserve the integrity of the useful signal, their sensitivity to the influence parameters must be sufficiently well-known and controlled, either in an empirical way, or by analytical laws. Whatever the purpose of their use or their operating mode, the thermal resistance behaviour must be apprehended in the most accurate way. In a previous study [1], we showed that the thermal drift of a silicon resistor doped with acceptors atoms could be described by an equation of the second order and that the two temperature coefficients (TCRs) have a strong dependence with the doping concentration. Following the same idea, we modelled the influence of the doping concentration on the temperature coefficients of a resistor doped with donor atoms. Knowing that there are differences between the laws controlling the mobility of the electrons and the holes, one can expect that the resistivity of this material shows different behaviour according to the doping type.

\section{Relation Resistivity-Mobility}

The expression of a silicon resistance according to the temperature $T$ is given by

$$
R(T)=R\left(T_{0}\right)\left[1+\alpha\left(T-T_{0}\right)+\beta\left(T-T_{0}\right)^{2}\right]
$$

where $T_{0}$ is a reference temperature; $\alpha$ and $\beta$ are the first and second order TCRs.

Knowing that $[1]$ :

$$
\frac{\Delta R(T)}{R\left(T_{0}\right)}=\frac{\Delta \rho(T)}{\rho\left(T_{0}\right)}=\frac{\Delta \mu\left(N_{D}, T\right)}{\mu\left(T_{0}\right)}
$$

where $\rho$ and $\mu$ are respectively the silicon resistivity and the carriers' mobility, it would be sufficient to model the carriers' mobility behaviour according to temperature and doping to obtain the expressions of the two coefficients (TCRs). So far, no analytical model allows us to describe this dependence. On the other hand, there are several empirical models describing the influence of doping and the temperature on the carriers mobility, among which we used those of Arora [2], Klaassen [3] and Dorckel [4] as is shown in the Appendix. In order to evaluate the total carrier mobility $\left(\mu_{i}\right.$ for electron and $\mu_{j}$ for hole), we use the Mathiessen's rule which approximates it at low longitudinal field as the sum of four term, which are the four contributions to the carrier mobility: Lattice mobility $\left(\mu_{i, L}\right)$; donor mobility $\left(\mu_{i, D}\right)$; acceptor mobility $\left(\mu_{i, A}\right)$ and electron-hole scattering mobility $\left(\mu_{i, j}\right)$, and includes temperature dependence $[5,6]$ :

$$
\frac{1}{\mu_{i}}=\frac{1}{\mu_{i, L}}+\frac{1}{\mu_{i, D}}+\frac{1}{\mu_{i, A}}+\frac{1}{\mu_{i, j}}
$$

Thus, using a limited development of the mobility's expressions $\mu=f\left(N_{D}, T\right)$ taken from these references, we used a method of parameters identification. This method is a term by term comparison of the mobility expression as a function of temperature, with Equation (2), for each concentration. Thus, we obtain the expression of $\alpha=f\left(N_{D}\right)$ and $\beta=f\left(N_{D}\right)$. 


\section{Results}

The obtained results allowed us to plot the curves in Figures 1 and 2. These figures show that for the three models, there is a strong similarity in the shape of the nonlinear curves $\alpha=f\left(N_{D}\right)$ and $\beta=f\left(N_{D}\right)$. Moreover, the shapes of these curves are similar to those published in [1], as shown in Figures 3 and 4; moreover the minimum values appear for a given doping.

The comparison between the results previously published for a P-type resistor (Figures 3 and 4) and those

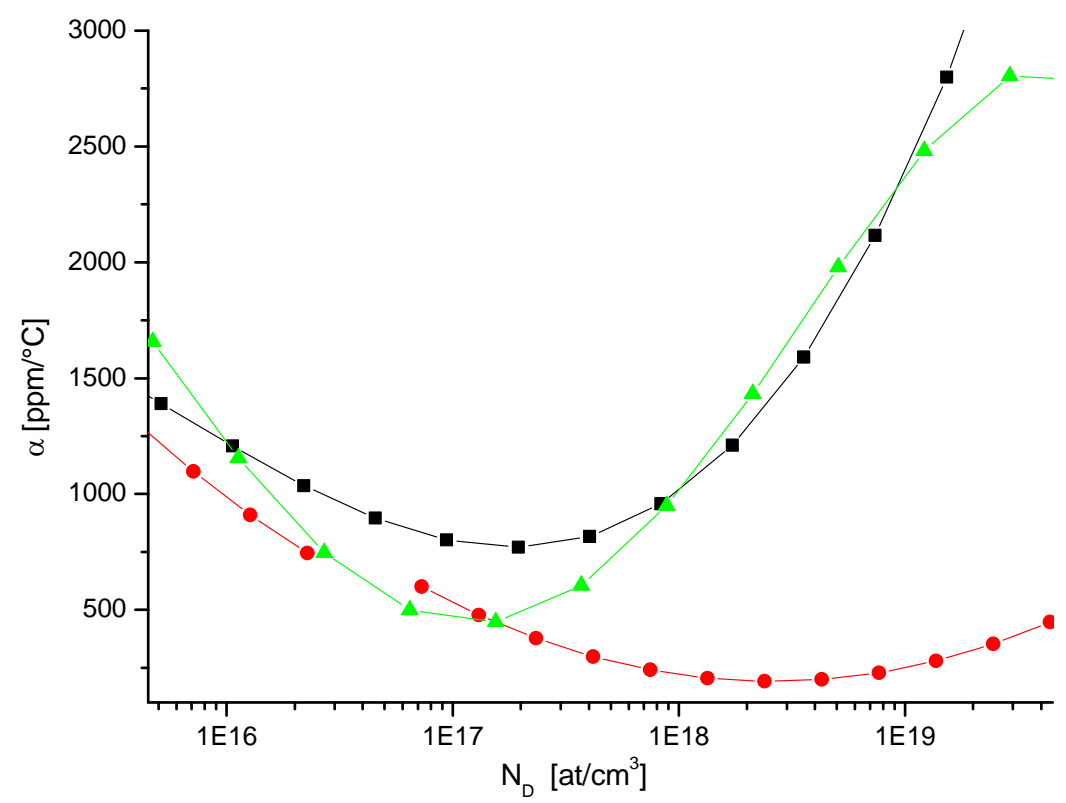

Figure 1. Variations of the first order thermal coefficient $\alpha$ as a function of doping concentration $N_{D}$. - Klaassen; $\alpha_{\min }=$ $445 \mathrm{ppm} /{ }^{\circ} \mathrm{C}$; - Arora $; \alpha_{\min }=770 \mathrm{ppm} /{ }^{\circ} \mathrm{C} ; \multimap$ Dorckel; $\alpha_{\min }=190 \mathrm{ppm} /{ }^{\circ} \mathrm{C}$.

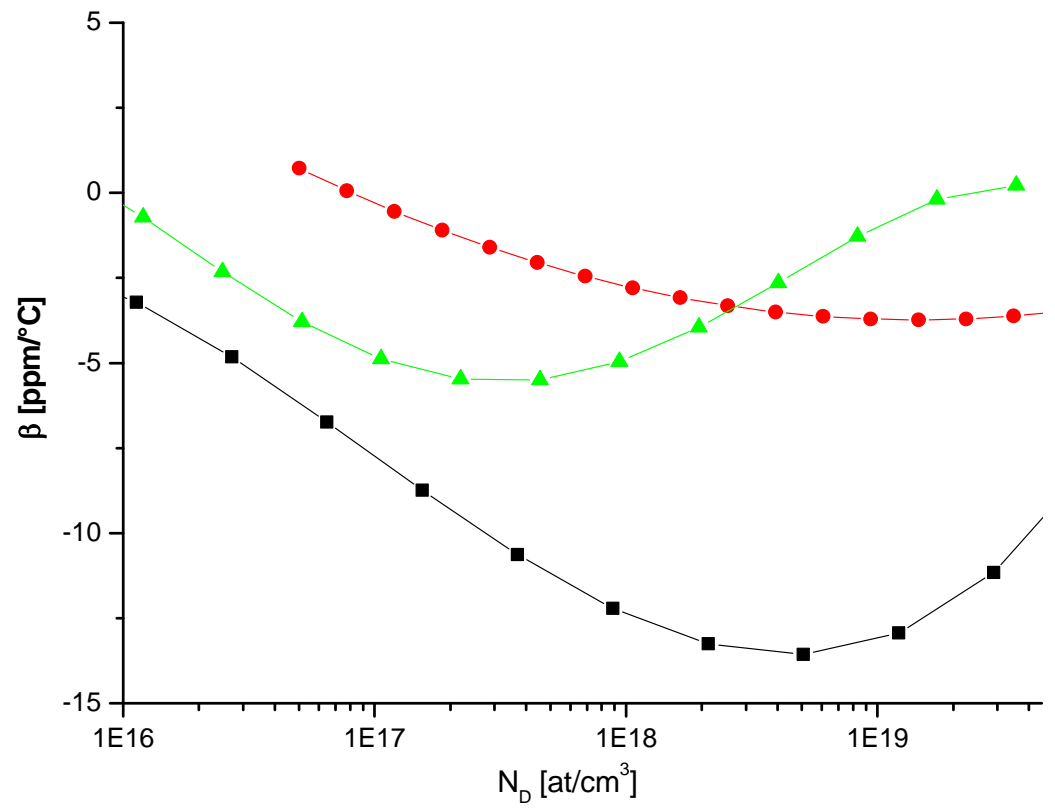

Figure 2. Variations of the second order thermal coefficient $\beta$ as a function of doping concentration $N_{D}$. $-\subset$ Dorckel; $\beta_{\text {min }}$ $=-3.80 \mathrm{ppm} /{ }^{\circ} \mathrm{C} ;-\mathrm{Klaassen} ; \boldsymbol{\beta}_{\min }=-5.00 \mathrm{ppm} /{ }^{\circ} \mathrm{C} ;-\operatorname{Arora} ; \beta_{\min }=-13.6 \mathrm{ppm} /{ }^{\circ} \mathrm{C}$. 


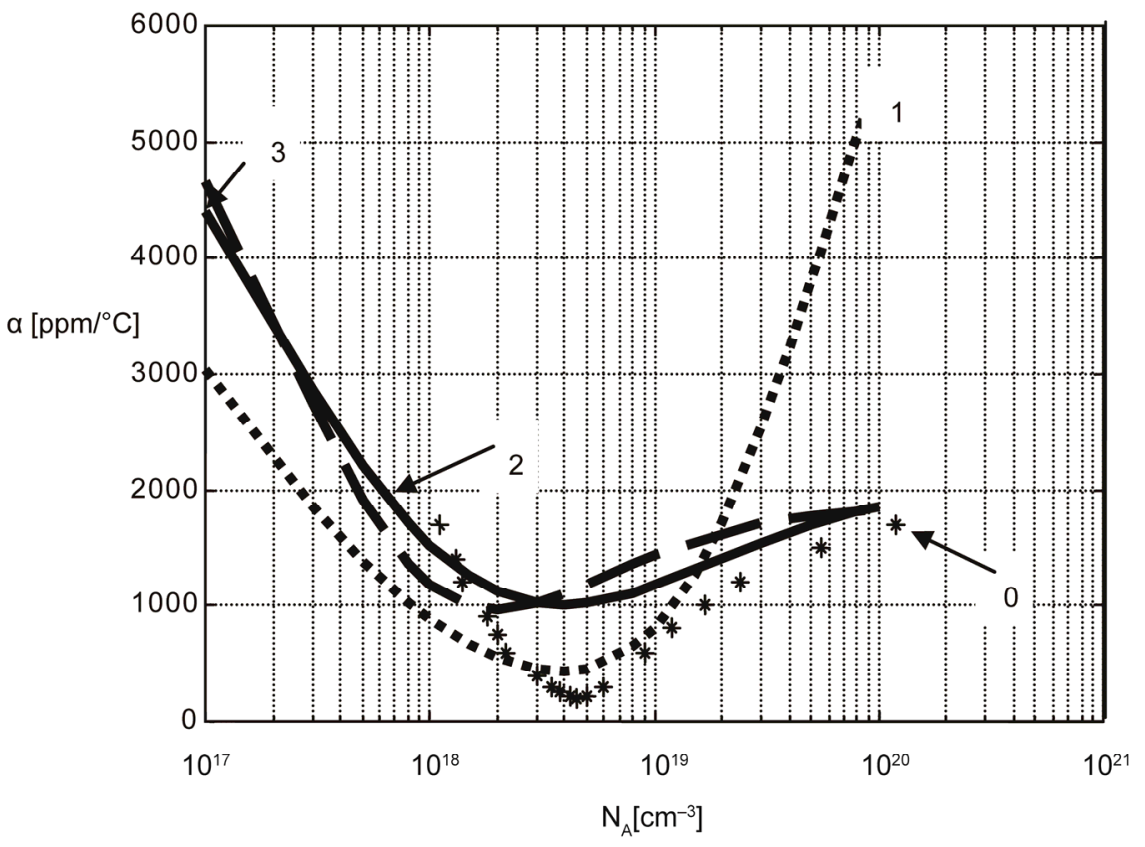

Figure 3. Variations of the first order thermal coefficient $\alpha$ as a function of doping concentration $N_{A}$ [1]. 0: Bullis [6] $\alpha_{\min }=$ $200 \mathrm{ppm} /{ }^{\circ} \mathrm{C} ; 1$ : Dorckel [4] $\alpha_{\min }=400 \mathrm{ppm} /{ }^{\circ} \mathrm{C} ; 2$ : Arora [2] $\alpha_{\min }=980 \mathrm{ppm} /{ }^{\circ} \mathrm{C} ; 3$ : Klaassen $[3] \alpha_{\min }=1000 \mathrm{ppm} /{ }^{\circ} \mathrm{C}$

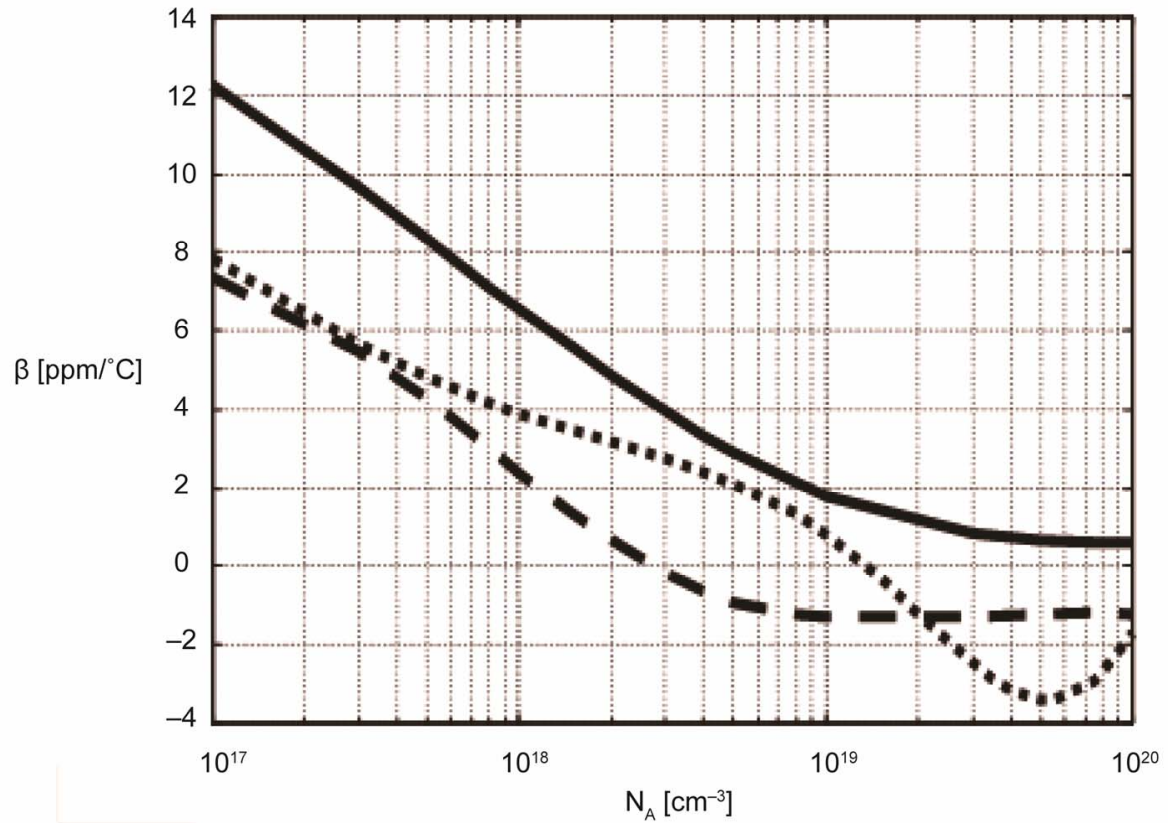

Figure 4. Variations of the second order thermal coefficient $\beta$ as a function of doping concentration $N_{A}[1]$.

(Figure 5 for the type P; Figure 6 for the type N). Since the minimum value for different models do not occur at the same doping concentration for the $\alpha$ parameter than for the $\beta$ one, then we have choose, for a same doping concentration, particular coefficients couples $\left(\alpha_{\min }, \beta\right)$.

These values are used to evaluate the relative resistance change $\Delta R / R$ as a function of the temperature variation $\Delta T$. The minimum values of the first and second order thermal coefficients were determined in order to predict the variation of the P-type and the N-type thermal drift resistance, for a given doping concentration.

Figure 5 shows clearly that for a couple $\alpha_{\min }\left(N_{A}\right)$ and $\beta_{\min }\left(N_{A}\right)$ for the P-type resistor thermal variations can be considered as linear in a large interval of temperature. Figure 6 shows that the N-type resistor thermal behavior is highly nonlinear, whatever the values of $\alpha_{\min }\left(N_{D}\right)$ and $\beta_{\min }\left(N_{D}\right)$. Therefore, these results may help the more complicated circuits to choose the resistors doping con 


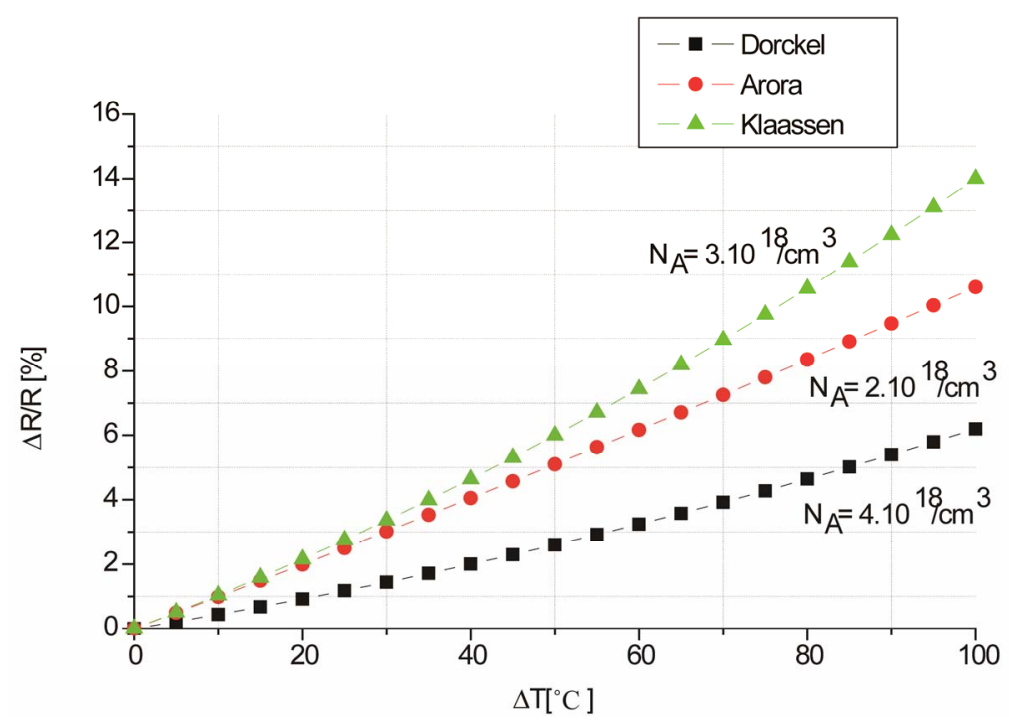

Figure 5. Variations of the relative resistance $\Delta R / R$ as a function of the temperature gradient $\Delta T$ for a particular couple $\left(\alpha_{\min }\left(N_{A}\right), \beta_{\min }\left(N_{A}\right)\right)$, for a given doping concentration.

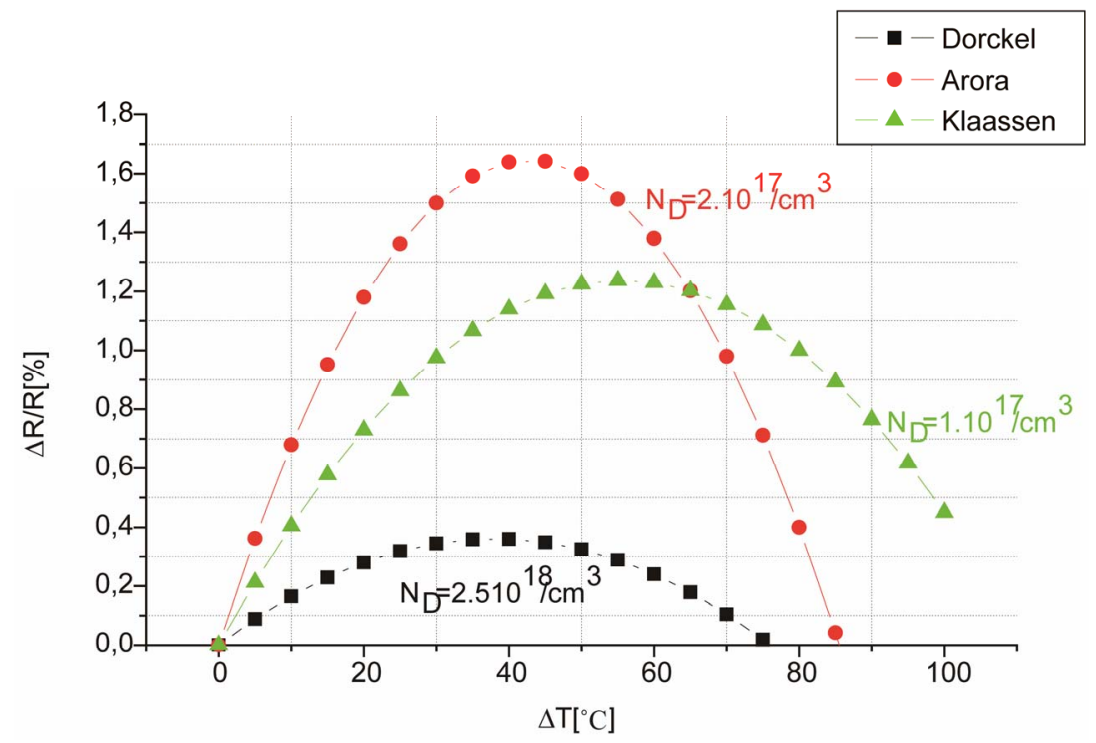

Figure 6. Variations of the relative resistance $(\Delta R / R)$ as a function of the temperature gradient $\Delta T$ for a particular couple $\left(\alpha_{\min }\left(N_{D}\right), \beta_{\min }\left(N_{D}\right)\right)$, for a given doping concentration.

centration used to obtain a minimum thermal drift depending on the desired application.

\section{Conclusion}

By using three models of carriers' mobility in silicon resistance doped with donor's atoms, we showed that the 1 st and the 2 nd order thermal coefficient can present a minimal value for a given doping. In addition, the comparison of the variations in a range limited of the temperature, between resistances of the two types $\mathrm{N}$ and $\mathrm{P}$, shows that their behaviour is similar. Indeed, there is a particular couple $\left(\alpha_{\min }\right.$ and $\left.\beta_{\min }\right)$ which allow the lineari zation of the curve $\Delta R / R=f(\Delta T)$ for a P-type resistor, but not for the type N. The obtained results can help the designer of more complex circuits, to choose the used resistors doping concentrations. Thereby, further enhance the performance of these devices in terms of thermal drift, according to the desired application.

\section{REFERENCES}

[1] A. Boukabache and P. Pons, "Doping Effects on Thermal Behaviour of Silicon Resistor," Electronics Letters, Vol. 38, No. 7, 2002, pp. 342-343. doi:10.1049/el:20020221

[2] N. D. Arora and D. J. Roulston, "Electron and Hole Mo- 
tilities in Silicon as a Function of Concentration and Temperature" IEEE Transactions on Electron Devices, Vol. 29, No. 2, 1982, pp. 292-295. doi:10.1109/T-ED.1982.20698

[3] D. B. M. Klaassen, "Unified Model for Device Simulation-I-II. Temperature Dependence of Carrier Mobility and Life Time A," Solid State Electronic, Vol. 35, No. 7, 1992, pp. 953-967.

[4] J. M. Dorckel and P. H. Leturcq, "Carrier Motilities in Silicon Semi-Empirically Related to Temperature, Doping and Injection Level," Solid State Electronic, Vol. 24,

\section{Appendix}

In this appendix, we describe several models of mobility's carrier used in the literature.

\section{N. D. ARORA et al. Mobility Model [2]}

$$
\begin{aligned}
\mu_{e}(N, T)= & 88 \times\left(\frac{T}{300}\right)^{-0.57} \\
+ & \frac{7.410^{8} T^{-2.23}}{1+0.698410^{17} \times N \times\left(\frac{T}{300}\right)^{-2.546}}
\end{aligned}
$$

where $T$ is the temperature in Kelvin and $N$ is the doping concentration

\section{B. M. Klaassen Mobility Model [3]}

In this model, the four contributions to the carrier mobility are taken into account, and the expression of the total mobility is:

With $P_{e}, G\left(P_{e}\right)$ and $F\left(P_{e}\right)$ are given by (2.2), (2.3) and (2.4):

$$
\begin{aligned}
P_{e}= & \frac{1.3610^{20}}{c} \times\left(\frac{m_{e}}{m_{0}}\right) \times\left(\frac{T}{300}\right)^{2} \\
G\left(P_{e}\right)= & 1-\frac{s_{1}+\left(\left(\frac{m_{0}}{m_{e}}\right) \times\left(\frac{T}{300}\right)\right)^{s_{4}}}{\left(\left(\left(\frac{m_{e}}{m_{0}}\right) \times\left(\frac{300}{T}\right)\right)^{s_{7}} P_{e}\right)^{s_{6}}} \\
& +\frac{s_{5}}{(()}
\end{aligned}
$$

No. 9, 1981, pp. 821-825. doi:10.1016/0038-1101(81)90097-6

[5] C. Lombardi, S. Manzini, A. Saporito and M. Vanzi, "A Physically Based Mobility Model for Numerical Simulation of Non Planar Devices," IEEE Transacitions Computer-Aided Design, Vol. 7, 1988, pp. 1164-1171.

[6] T. T. Mnatsakanov, M. E. Levinshtein, L. I. Pomortseva1 and S. N. Yurkov1, "Carrier Mobility Model for Simulation of SiC-Based Electronic Devices," Semiconductor Science and Technology, Vol. 17, No. 9, 2002, pp. 974 977.

$$
F\left(P_{e}\right)=\frac{r_{1} P_{e}^{r_{6}}+r_{2}+r_{3} \frac{m_{1}}{m_{2}}}{P_{e}^{r_{6}}+r_{4}+r_{5} \frac{m_{2}}{m_{1}}}
$$

where $m$ and $m_{0}$ are the effective and the free carrier mass respectively, $T$ is the temperature in Kelvin, with $m_{1}$ and $m_{2}$ the mass of the primary and secondary scatters respectively. The model parameters for the majority electron and hole mobility given by Equation (2.1) are summarized in Table 2.1. The numerical value of the constants $s_{i}$ and $r_{i}$ are given in Table 2.2.

\section{J. M. Dorckel et al. Mobility Model [4]}

Lattice mobility $\mu_{L}$ which is connected to scattering due to acoustic phonons

$$
\mu_{L}=\mu_{L 0} \times\left(\frac{T}{300}\right)^{-\alpha}
$$

Impurity mobility $\mu_{I}$ which is connected to the interactions between the carriers and the ionized impurities

$$
\mu_{I}=\frac{A T^{1.5}}{N}\left[\ln \left(1+\frac{B T^{2}}{N}\right)-\frac{B T^{2}}{N+B T^{2}}\right]^{-1}
$$

Carrier-carrier scattering mobility $\mu_{c c s}$ which becomes very influential when carriers of both types are at high concentrations

$$
\mu_{c c s}=\frac{210^{7} T^{1.5}}{\sqrt{p n}}\left[\ln \left(1+\frac{8.2810^{8} T^{2}}{\sqrt[3]{p n}}\right)\right]^{-1}
$$

$$
\mu_{e}(N, T)=\frac{1}{\frac{1}{\mu_{\text {max }}}+\frac{\mu_{\text {max }}-\mu_{\text {min }}}{\mu_{\text {max }}^{2} \times\left(\frac{N r e f}{N_{D}}\right)^{\alpha 1}+\mu_{\text {max }} \mu_{\text {min }}}+\frac{\mu_{\text {max }}-\mu_{\min }}{\mu_{\text {max }}^{2} \times\left(\frac{N r e f}{N_{D}}\right)^{\alpha 1}+\mu_{\text {max }} \mu_{\text {min }}}(1+G(P e)+F(P e))}
$$


Table 2.1. Model parameters for the majority electron mobility (As and P) and majority hole mobility (B) given by Equation (2.1).

\begin{tabular}{cccc}
\hline Parameters & As & P & B \\
\hline$\mu_{\max }\left(\mathrm{cm}^{2} \cdot \mathrm{V}^{-1} \cdot \mathrm{S}^{-1}\right)$ & 1417 & 1414 & 470.5 \\
$\mu_{\min }\left(\mathrm{cm}^{2} \cdot \mathrm{V}^{-1} \cdot \mathrm{S}^{-1}\right)$ & 52.2 & 68.5 & 44.9 \\
$\mu_{1}\left(\mathrm{~cm}^{2} \cdot \mathrm{V}^{-1} \cdot \mathrm{S}^{-1}\right)$ & 43.4 & 56.1 & 29 \\
Nref, $1\left(\mathrm{~cm}^{-3}\right)$ & $9.68 \times 10^{16}$ & $9.2 \times 10^{16}$ & $2.23 \times 10^{17}$ \\
Nref, $2\left(\mathrm{~cm}^{-3}\right)$ & $3.43 \times 10^{20}$ & $3.41 \times 10^{20}$ & $6.1 \times 10^{20}$ \\
& 0.68 & 0.71 & 0.719 \\
\hline
\end{tabular}

Table 2.2. Numerical values for the constants $s_{i}$ and $r_{i}$.

\begin{tabular}{ccc}
\hline$i$ & $s_{i}$ & $r_{i}$ \\
\hline 1 & 0.89233 & 0.7643 \\
2 & 0.41372 & 2.2999 \\
3 & 0.19778 & 6.5502 \\
4 & 0.25227 & 2.367 \\
5 & 0.005978 & -0.01552 \\
6 & 1.80618 & 0.6478 \\
7 & 0.72169 & \\
\hline
\end{tabular}

Table 3.1. Model parameters values.

\begin{tabular}{cc}
\hline Parameters & As \\
\hline$\mu_{L 0}\left(\mathrm{~cm}^{2} \cdot \mathrm{V}^{-1} \cdot \mathrm{S}^{-1}\right)$ & 1430 \\
$\mathrm{~A}\left(\mathrm{~cm}^{2} \cdot \mathrm{V}^{-1} \cdot \mathrm{S}^{-1} \cdot \mathrm{K}^{-1.5}\right)$ & $4.611 \times 10^{17}$ \\
$\mathrm{~B}\left(\mathrm{~cm}^{-3} \cdot \mathrm{K}^{-2}\right)$ & $1.52 \times 10^{15}$ \\
& 2.2 \\
\hline
\end{tabular}

$$
\mu_{c c s}=\frac{210^{7} T^{1.5}}{\sqrt{p n}}\left[\ln \left(1+\frac{8.2810^{8} T^{2}}{\sqrt[3]{p n}}\right)\right]^{-1}
$$

Mixed scattering mobility $\mu_{\text {Iccs }}$

$$
\mu_{\text {Iccs }}=\left(\frac{1}{\mu_{I}}+\frac{1}{\mu_{c c s}}\right)^{-1}
$$

where $T$ is the temperature in Kelvin and $N$ is the doping concentration, $\mu_{L}$ is the lattice mobility, $\mu_{I}$ is the impurity mobility, $\mu_{c s s}$ is the carrier inter-action mobility and $\mu_{\text {Icss }}$ is the carrier-impurity inter-action mobility. The model parameters values are summarized in Table 3.1. 